\title{
SPLITTING OF 3-MANIFOLDS AND RIGIDITY OF AREA-MINIMISING SURFACES
}

\author{
MARIO MICALLEF AND VLAD MORARU \\ (Communicated by Michael Wolf)
}

\begin{abstract}
In this paper we modify an argument of Bray, Brendle and Neves to prove an area comparison result (Theorem 2) for certain totally geodesic surfaces in 3-manifolds with a lower bound on the scalar curvature.

This theorem is a variant of a comparison theorem (Theorem 3.2 (d) in the 1978 paper) of Heintze and Karcher for minimal hypersurfaces in manifolds of nonnegative Ricci curvature. Our assumptions on the ambient manifold are weaker, but the assumptions on the surface are considerably more restrictive.

We then use our comparison theorem to provide a unified proof of various splitting theorems for 3-manifolds with lower bounds on the scalar curvature that were first proved by Cai and Galloway; Bray, Brendle and Neves; and Nunes.
\end{abstract}

\section{Introduction AND STATEMENT OF MAIN RESUlts}

In 14, Corollary 3.6.1, Simons observed that there are no closed, stable, minimal, 2-sided hypersurfaces in a manifold of positive Ricci curvature. This is a variant of the classical Synge lemma. An easy, but unstated, extension of Simons's observation is that a closed, stable, minimal, 2 -sided hypersurface $\Sigma$ in a manifold $M$ of nonnegative Ricci curvature is necessarily totally geodesic and the normal Ricci curvature of $M$ must vanish all along $\Sigma$. The simplest such example is $\Sigma \times(-\varepsilon, \varepsilon), \varepsilon>0$, with the product metric $g+d t^{2}$, where the metric $g$ on $\Sigma$ has nonnegative Ricci curvature. However, the existence of a closed, stable, minimal, 2-sided hypersurface $\Sigma$ in a manifold $M$ of nonnegative Ricci curvature does not imply that the metric of $M$ near $\Sigma$ must split as $g+d t^{2}$. Indeed, the metric $\left(1-t^{4}\right) g+d t^{2}$ on $\Sigma \times(-\varepsilon, \varepsilon), 1>\varepsilon>0$, has nonnegative Ricci curvature if $g$ has nonnegative Ricci curvature and $\Sigma \times\{0\}$ is stable 1 However, note that in this example, $\Sigma \times\{0\}$ does not minimise area, not even locally.

So, one might surmise whether the existence of a closed, area-minimising, 2-sided hypersurface $\Sigma$ in a manifold $M$ of nonnegative Ricci curvature implies that the metric of $M$ near $\Sigma$ must split as $g+d t^{2}$. This is indeed the case and follows from a special case of Theorem 3.2 (d) in [10, where Heintze and Karcher prove that the exponential map of the normal bundle $\Sigma \times \mathbb{R}$ of $\Sigma$ in $M$ is volume nonincreasing when $M$ has nonnegative Ricci curvature, $\Sigma$ is two-sided and minimal in $M$ and $\Sigma \times \mathbb{R}$ is equipped with the product metric. Anderson extended this result to areaminimising integral currents of codimension 1 in a compact manifold of nonnegative

Received by the editors March 10, 2012 and, in revised form, December 29, 2012.

2010 Mathematics Subject Classification. Primary 49Q05, 53C24; Secondary 26D10.

${ }^{1}$ If $g$ is the round metric on the unit sphere $S^{n}$, then it is easy to embed this example, for $\varepsilon$ sufficiently small, into a metric on $S^{n+1}$ of nonnegative sectional curvature. 
Ricci curvature; see Theorem 3 in [1]. Anderson then used this theorem, together with an existence result for area-minimising hypersurfaces, to obtain a different proof of the splitting theorem in [6] of Cheeger and Gromoll in the compact case; see Corollary 3 in 1 .

It is easy to construct examples of totally geodesic, area-minimising hypersurfaces in manifolds of positive scalar curvature. Therefore, positive Ricci curvature in Simons's result cannot be relaxed to positive scalar curvature. Nevertheless, in the well-known paper [12, Schoen and Yau obtained topological restrictions on an oriented, two-sided, stable, minimal surface $\Sigma$ in a 3 -manifold $M$ whose scalar curvature $R$ is positive. In particular, they proved that the genus of $\Sigma$ must be zero. Soon after, Fischer-Colbrie and Schoen studied the case $R \geqslant 0$ and proved in [7] that, in this case, the genus of $\Sigma$ must be zero or one, and if it is one, then $\Sigma$ is totally geodesic and flat and both the normal Ricci curvature of $M$ and $R$ vanish all along $\Sigma$.

A closer look at the proof of Schoen-Yau reveals that a lower bound on the scalar curvature $R$ of the 3 -manifold $M$ provides a bound on the area of the stable minimal surface $\Sigma$. More precisely,

(1) if $R \geqslant 2$, then the area of $\Sigma$ is bounded above by $4 \pi$; and

(2) if $R \geqslant-2$, then the area of $\Sigma$ is bounded below by $4 \pi(\gamma-1)$, where $\gamma$ is the genus of $\Sigma$.

To our knowledge, these bounds first appeared in [13. (These bounds also appear in the literature of general relativity. See, for example, [8].) Easy examples show that no area bounds are possible for stable minimal tori in flat three-dimensional tori. If the area bound in (1) or (2) is attained and $R_{0}:=\min _{x \in M} R(x)$, then an analysis similar to that used by Fischer-Colbrie and Schoen in the case of genus one yields that

(i) the surface is totally geodesic,

(ii) $R$ is equal to $R_{0}$ all along $\Sigma$ and

(iii) the normal Ricci curvature of $M$ vanishes all along $\Sigma$.

It follows that

(iv) the Gauss curvature $K$ of $\Sigma$ is identically equal to $\frac{1}{2} R_{0}$.

The obvious examples in which the area bound (1) or (2) is attained are provided by $\Sigma \times(-\varepsilon, \varepsilon), \varepsilon>0$, with the product metric $g+d t^{2}$, where $g$ has constant Gauss curvature equal to $\frac{1}{2} R_{0}$. A natural question is whether these are the only examples in which equality is attained in (1) and (2). As in the case of nonnegative Ricci curvature mentioned earlier, this does not hold. Indeed, the scalar curvature $R$ of the metric $\left(1-t^{4}\right) g+d t^{2}$ satisfies $R \geqslant R_{0}$ and $\Sigma \times\{0\}$ is stable but, once again, does not minimise area, not even locally.

So again, one surmises whether the metric near an area-minimising closed surface $\Sigma$ which satisfies (i), (ii) and (iii), in a 3-manifold $M$ whose scalar curvature $R$ is greater than or equal to $R_{0}$, splits. The examples of totally geodesic, areaminimising hypersurfaces in manifolds of positive scalar curvature that come easily to mind do not satisfy condition (ii).

To make use of the area-minimising property, one has to perturb $\Sigma$ in a way which decreases its area. When the Ricci curvature of $M$ is nonnegative, Heintze and Karcher (and, to second order, Simons) showed that this is achieved by surfaces that are equidistant from $\Sigma$. However, in our case we do not have information on the 
Ricci tensor away from $\Sigma$, and so this is not a suitable perturbation. We shall see that the right thing to do is to move $\Sigma$ so that it still has constant mean curvature. This turns out to be possible by Proposition 3.2 in [3], which essentially asserts the existence of a one-parameter family of constant mean curvature surfaces in a neighborhood of a surface $\Sigma$ which satisfies (i) and (iii); see also [2]. A detailed proof of the following statement, based on the implicit function theorem, can be found in [11]. See also [2] and [4].

Proposition 1. Let $\nu$ be a unit normal field on an oriented, two-sided surface $\Sigma$ immersed in a 3-manifold $M$. If $\Sigma$ satisfies (i) and (iii), then there exists $\varepsilon>0$ and a smooth function $w: \Sigma \times(-\varepsilon, \varepsilon) \rightarrow \mathbb{R}$ such that, for all $t \in(-\varepsilon, \varepsilon)$, the surfaces

$$
\Sigma_{t}:=\left\{\exp _{x}(w(x, t) \nu(x)): x \in \Sigma\right\}
$$

have constant mean curvature $H(t)$. Moreover, we have

$$
w(x, 0)=0,\left.\quad \frac{\partial}{\partial t} w(x, t)\right|_{t=0}=1, \quad \text { and } \quad \int_{\Sigma}(w(\cdot, t)-t) d A=0
$$

for all $x \in \Sigma$ and $t \in(-\varepsilon, \varepsilon)$.

We shall prove the following theorem, which, in light of the preceding discussion, is the optimal analogue of the Heintze-Karcher Theorem 3.2(d) in [10] in the context of 3-manifolds with lower bounds on scalar curvature. The proof relies heavily on the Gauss-Bonnet Theorem, and so it is not at all clear how it may be generalised to dimension 4 or higher.

Theorem 2. Let $M$ be a 3-manifold and let $\Sigma \subset M$ be an immersed, 2-sided, closed surface. Denote by $R$ the scalar curvature of $M$, and let $R_{0}:=\min _{x \in M} R(x)$. Suppose that $\Sigma$ has the following properties:

(i) $\Sigma$ is totally geodesic,

(ii) $R$ is equal to $R_{0}$ all along $\Sigma$ and

(iii) the normal Ricci curvature of $M$ vanishes all along $\Sigma$.

Let $\Sigma_{t}$ and $\varepsilon$ be as in Proposition 1 and denote by $A(t)$ the area of $\Sigma_{t}$. (In particular, $A(0)$ is the area of $\Sigma$.) Then there exists $0<\delta<\varepsilon$ such that

$$
\text { for }|t|<\delta, \quad A(t) \leqslant A(0) .
$$

Moreover, $\Sigma$ has constant Gauss curvature equal to $\frac{1}{2} R_{0}$, and therefore, by GaussBonnet, $A(0)=\frac{8 \pi}{\left|R_{0}\right|}|\gamma-1|$ if $R_{0}$ is nonzero.

Theorem 2 is the key ingredient that we use to provide a unified and more elementary proof of the following splitting and rigidity theorem (Theorem 3), the three cases of which were separately proved, using different techniques, in [3], 5] and [1]. Indeed, our research for Theorem 2 was sparked by a lecture that A. Neves gave at the University of Warwick in which he presented the results in [3. An interesting connection with the Yamabe problem is made in [11, which appeared on the arXiv preprint website while the present paper was being written.

Theorem 3. Let $(M, g)$ be a complete Riemannian 3-manifold with scalar curvature $R$ and let $R_{0}:=\min _{x \in M} R(x)$. Assume that $M$ contains a closed, embedded, oriented, two-sided, area-minimizing surface $\Sigma$. 
(1) Suppose that $R_{0}=2$ and that the area of $\Sigma$ is equal to $4 \pi$. Then $\Sigma$ has genus zero and it has a neighbourhood which is isometric to the product $g_{1}+d t^{2}$ on $S^{2} \times(-\delta, \delta)$, where $g_{1}$ is the metric on the Euclidean two-sphere of radius 1 .

(2) Suppose that $R_{0}=0$ and that $\Sigma$ has genus one. Then $\Sigma$ has a neighbourhood which is flat and isometric to the product $g_{0}+d t^{2}$ on $T^{2} \times(-\delta, \delta)$, where $g_{0}$ is a flat metric on the torus $T^{2}$.

(3) Suppose that $R_{0}=-2$ and that $\Sigma$ has genus $\gamma \geqslant 2$ and area equal to $4 \pi(\gamma-1)$. Then $\Sigma$ has a neighbourhood which is isometric to the product $g_{-1}+d t^{2}$ on $\Sigma \times(-\delta, \delta)$, where $g_{-1}$ is a metric of constant Gauss curvature equal to -1 on $\Sigma$.

\section{THE PROOFS}

Proof of Theorem 2. With the notation as in Proposition 1, let

$$
f_{t}(x):=\exp _{x}(w(x, t) \nu(x)), \quad x \in \Sigma, t \in(-\varepsilon, \varepsilon) .
$$

Thus, $f_{0}=: f$ is the given totally geodesic embedding. The lapse function $\rho_{t}: \Sigma \rightarrow$ $\mathbb{R}$ is defined by

$$
\rho_{t}(x):=\left\langle\nu_{t}(x), \frac{\partial}{\partial t} f_{t}(x)\right\rangle
$$

where $\nu_{t}$ is a unit normal to $\Sigma_{t}$, chosen so as to be continuous in $t$. It satisfies the following Jacobi equation (cf. 9], eq. (1.2)):

$$
H^{\prime}(t)=-\Delta_{\Sigma_{t}} \rho_{t}-\left(\operatorname{Ric}\left(\nu_{t}, \nu_{t}\right)+\left\|B_{t}\right\|^{2}\right) \rho_{t},
$$

where $B_{t}$ is the second fundamental form of $\Sigma_{t}$ and $(\cdot)^{\prime}:=\frac{\partial}{\partial t}(\cdot)$. Since $\rho_{0}(x)=1$ for all $x \in \Sigma$, we can assume, by decreasing $\varepsilon$ if necessary, that $\rho_{t}(x)>0$ for all $x \in \Sigma$. So we can divide (2) by $\rho_{t}$ and, using the Gauss equation

$$
\operatorname{Ric}(\nu, \nu)=\frac{1}{2} R-K+\frac{1}{2} H^{2}-\frac{1}{2}\|B\|^{2},
$$

(2) becomes

$$
H^{\prime}(t) \frac{1}{\rho_{t}}=-\frac{1}{\rho_{t}} \Delta_{\Sigma_{t}} \rho_{t}-\frac{1}{2} R_{t}+K_{t}-\frac{1}{2} H(t)^{2}-\frac{1}{2}\left\|B_{t}\right\|^{2},
$$

where $R_{t}(x):=R\left(f_{t}(x)\right)$ and $K_{t}(x):=K\left(f_{t}(x)\right)$ is the Gauss curvature of $\Sigma_{t}$ at $f_{t}(x)$. We now make essential use of the hypotheses (i), (ii) and (iii), which, via the Gauss equation (3), imply that

$$
K_{0} \equiv \frac{1}{2} R_{0} \leqslant \frac{1}{2} R_{t}(x) \quad \forall x \in \Sigma, t \in(-\varepsilon, \varepsilon),
$$

and therefore (4) can be rewritten as

$$
\begin{aligned}
H^{\prime}(t) \frac{1}{\rho_{t}} & =-\frac{1}{\rho_{t}} \Delta_{\Sigma_{t}} \rho_{t}+\frac{1}{2}\left(R_{0}-R_{t}\right)+\left(K_{t}-K_{0}\right)-\frac{1}{2} H(t)^{2}-\frac{1}{2}\left\|B_{t}\right\|^{2} \\
& \leqslant-\frac{1}{\rho_{t}} \Delta_{\Sigma_{t}} \rho_{t}+\left(K_{t}-K_{0}\right) .
\end{aligned}
$$

We integrate (6) over $\Sigma_{t}$ (by parts in the first term on the right), and, keeping in mind that $H$ does not depend on $x$ (hence the importance of using a constant mean 
curvature perturbation), we obtain

$$
H^{\prime}(t) \int_{\Sigma} \frac{1}{\rho_{t}} d A_{t} \leqslant-\int_{\Sigma} \frac{\left\|\nabla_{t} \rho_{t}\right\|^{2}}{\rho_{t}^{2}} d A_{t}+\int_{\Sigma}\left(K_{t}-K_{0}\right) d A_{t}
$$

$$
\leqslant 4 \pi(1-\gamma)-K_{0} A(t),
$$

where $d A_{t}$ denotes the area element of $\Sigma_{t}$ with respect to $f_{t}^{*} g$ and we have used the Gauss-Bonnet Theorem in the last inequality.

Claim 1. There exists a positive real number $\delta<\varepsilon$ such that $H(t) \leqslant 0$ for all $t \in[0, \delta)$.

Proof of Claim 1. There are three cases to consider.

Case 1. $R_{0}>0$. By scaling, we can arrange $R_{0}=2$. Then by (15), we have that $K_{0} \equiv 1$, and $\Sigma$ has genus zero and $A(0)=4 \pi$. Therefore inequality (7) becomes

$$
\begin{aligned}
H^{\prime}(t) \int_{\Sigma} \frac{1}{\rho_{t}} d A_{t} & \leqslant 4 \pi-A(t)=A(0)-A(t)=-\int_{0}^{t} A^{\prime}(s) d s \\
& =-\int_{0}^{t}\left\{H(s) \int_{\Sigma} \rho_{s} d A_{s}\right\} d s,
\end{aligned}
$$

where, in the last equality, we have used the first variation of area formula,

$$
A^{\prime}(t)=\int_{\Sigma} H(t) \rho_{t} d A_{t}=H(t) \int_{\Sigma} \rho_{t} d A_{t} .
$$

Let $\phi(t):=\int_{\Sigma} \frac{1}{\rho_{t}} d A_{t}$ and $\xi(t):=\int_{\Sigma} \rho_{t} d A_{t}$. Since $\phi$ is strictly positive for all $t \in(-\varepsilon, \varepsilon)$, inequality (8) becomes

$$
H^{\prime}(t) \leqslant-\frac{1}{\phi(t)} \int_{0}^{t} H(s) \xi(s) d s .
$$

As mentioned above, $\rho_{0} \equiv 1$ and, by continuity, we may assume that $\frac{1}{2}<\rho_{t}(x)<$ $2 \forall t \in(-\varepsilon, \varepsilon)$ and $x \in \Sigma$. Integrating over $\Sigma_{t}$ yields $\frac{1}{2} A(t)<\xi(t)<2 A(t)$. On the other hand, by choosing $\varepsilon>0$ small enough, we may assume that $\frac{1}{2} A(0)<A(t)<$ $2 A(0)$, and hence $\frac{1}{4} A(0)<\xi(t)<4 A(0) \forall t \in(-\varepsilon, \varepsilon)$. A similar argument holds for $\phi(t)$. In particular, we have

$$
\frac{1}{\phi(t)}<\frac{4}{A(0)} \quad \text { and } \quad \xi(t)<4 A(0), \forall t \in(-\varepsilon, \varepsilon) .
$$

Suppose, for a contradiction, that there exists $t_{+} \in(0, \delta)$ such that $H\left(t_{+}\right)>0$. By continuity, $\exists t_{-} \in\left[0, t_{+}\right)$such that $H\left(t_{-}\right) \leqslant H(t) \forall t \in\left[0, t_{+}\right]$. Note that by (10) we must have $H\left(t_{-}\right)<0$. By the mean value theorem, $\exists t_{1} \in\left(t_{-}, t_{+}\right)$such that

$$
H^{\prime}\left(t_{1}\right)=\frac{H\left(t_{+}\right)-H\left(t_{-}\right)}{t_{+}-t_{-}} .
$$

So, by (10) and (11), we have

$$
\frac{H\left(t_{+}\right)-H\left(t_{-}\right)}{t_{+}-t_{-}}=H^{\prime}\left(t_{1}\right) \leqslant-\frac{4}{A(0)} H\left(t_{-}\right)(4 A(0)) t_{1} .
$$

It follows that

$$
H\left(t_{+}\right) \leqslant H\left(t_{-}\right)\left(1-16 \delta^{2}\right)
$$

which is a contradiction if $0<\delta<\frac{1}{4}$ because $H\left(t_{+}\right)>0$ and $H\left(t_{-}\right)<0$. 
Case 2. $R_{0}=0$. By (5), we have that $K_{0} \equiv 0$ and $\Sigma$ has genus one. So, inequality (17) becomes $H^{\prime}(t) \leqslant 0 \forall t \in[0, \varepsilon)$, and therefore, since $H(0)=0, H(t) \leqslant 0 \forall t \in$ $[0, \varepsilon)$.

Case 3. $R_{0}<0$. By scaling, we can arrange $R_{0}=-2$. Then by (5), we have that $K_{0} \equiv-1$, and $\Sigma$ has genus $\gamma>1$ and $A(0)=4 \pi(\gamma-1)$. Therefore inequality (7) becomes

$$
\begin{aligned}
H^{\prime}(t) \int_{\Sigma} \frac{1}{\rho_{t}} d A_{t} & \leqslant A(t)-A(0)=\int_{0}^{t} A^{\prime}(s) d s \\
& =\int_{0}^{t}\left\{H(s) \int_{\Sigma} \rho_{s} d A_{s}\right\} d s .
\end{aligned}
$$

Assume, for a contradiction, that there exists $t_{0} \in(0, \delta)$ such that $H\left(t_{0}\right)>0$. Let

$$
I:=\left\{t \in\left[0, t_{0}\right]: H(t) \geqslant H\left(t_{0}\right)\right\}
$$

Claim 2. $\inf I=0$.

Proof of Claim 2. Let $t^{*}:=\inf I$ and assume, for a contradiction, that $t^{*}>0$. By the mean value theorem, $\exists t_{1} \in\left(0, t^{*}\right)$ such that

$$
H\left(t^{*}\right)=H^{\prime}\left(t_{1}\right) t^{*},
$$

since $H(0)=0$. From (12), (11) and (13) we have

$$
\begin{aligned}
H\left(t^{*}\right) & \leqslant \frac{t^{*}}{\phi\left(t_{1}\right)} \int_{0}^{t_{1}} H(s) \xi(s) d s \\
& \leqslant \frac{t^{*}}{\phi\left(t_{1}\right)} \int_{0}^{t_{1}} H\left(t^{*}\right) \xi(s) d s \leqslant \frac{4 t^{*}}{A(0)} H\left(t^{*}\right)\left(4 A(0) t_{1}\right) \\
& <16 H\left(t^{*}\right) \delta^{2},
\end{aligned}
$$

which is a contradiction if $\delta<\frac{1}{4}$, and Claim 2 has been proved.

Since $\inf I=0$, it follows from the definition of $I$ that $H(0) \geqslant H\left(t_{0}\right)$, and since, by assumption, $H\left(t_{0}\right)>0$, we conclude that $H(0)>0$. This contradicts the hypothesis that $\Sigma$ is totally geodesic, and the proof of Claim 1 is complete.

We can now easily complete the proof of Theorem 2. We have that $H(t) \leqslant 0 \forall t \in$ $[0, \delta)$, and therefore (9) implies that $A^{\prime}(t) \leqslant 0$. Hence $A(t) \leqslant A(0) \forall t \in[0, \delta)$. We can argue similarly for $t \in(-\delta, 0]$ to complete the proof of Theorem 2 ,

The proof of Theorem 3 now follows easily by a slight variation of arguments that appear in 3 and [11].

Proof of Theorem 3. The conclusion of Theorem 2 and the assumption that $\Sigma$ is area-minimising imply that, for the CMC family of surfaces $\Sigma_{t}$ provided by Proposition 1. $A(t)=A(0) \forall t \in(-\delta, \delta)$. In particular, each $\Sigma_{t}$ is area-minimising and, if $\gamma \neq 1$, the area of each $\Sigma_{t}$ is equal to $4 \pi|\gamma-1|$. It follows from (i) in the Introduction that each $\Sigma_{t}$ is totally geodesic. This holds when $\gamma=1$ as well. Equation (2) then tells us that the lapse function $\rho_{t}$ is harmonic and therefore is constant on $\Sigma_{t}$; i.e. $\rho_{t}$ is a function of $t$ only. 
Claim 3. The vector field $\nu_{t}$ is parallel.

Proof of Claim 3. $\Sigma_{t}$ is totally geodesic, and therefore $\nabla_{\frac{\partial f_{t}}{\partial x^{i}}} \nu_{t}=0$.

$$
\begin{aligned}
0=\frac{\partial}{\partial x^{i}} \rho_{t} & =\left\langle\nabla_{\frac{\partial f_{t}}{\partial x^{i}}} \nu_{t}, \frac{\partial f_{t}}{\partial t}\right\rangle+\left\langle\nu_{t}, \nabla_{\frac{\partial f_{t}}{\partial x^{i}}} \frac{\partial f_{t}}{\partial t}\right\rangle \\
& =\left\langle\nu_{t}, \nabla_{\frac{\partial f_{t}}{\partial x^{i}}} \frac{\partial f_{t}}{\partial t}\right\rangle \quad\left(\Sigma_{t} \text { is totally geodesic }\right) \\
& =\frac{\partial}{\partial t}\left\langle\nu_{t}, \frac{\partial f_{t}}{\partial x^{i}}\right\rangle-\left\langle\nabla_{\frac{\partial f_{t}}{\partial t}} \nu_{t}, \frac{\partial f_{t}}{\partial x^{i}}\right\rangle \\
& =-\left\langle\nabla_{\frac{\partial f_{t}}{\partial t}} \nu_{t}, \frac{\partial f_{t}}{\partial x^{i}}\right\rangle
\end{aligned}
$$

Hence $\nabla_{\frac{\partial f_{t}}{\partial t}} \nu_{t}=0$. This, together with the fact that $\Sigma_{t}$ is totally geodesic, implies that the vector field $\nu_{t}$ is parallel.

It follows that the integral curves of $\nu_{t}$ are geodesics and that the flow $\Phi$ of $\nu_{t}$ is just the exponential map, i.e. $\Phi(t, x)=\exp _{x}(t \nu(x)) \forall x \in \Sigma$. Furthermore, since $\nu_{t}$ is, in particular, a Killing field, this exponential map $\exp _{(\cdot)}(t \nu(\cdot))$ is an isometry for all $t \in(-\delta, \delta)$. In other words, if $g_{\Sigma}$ is the restriction of $g$ to $\Sigma$, then the exponential map of the $\delta$-neighbourhood $\Sigma \times(-\delta, \delta)$ of the zero section of the normal bundle of $\Sigma$ in $M$ with the metric $g_{\Sigma}+d t^{2}$ is an isometry onto its image.

Remark 4. It is straightforward to show that $w(x, t) \equiv t$ and that $\rho_{t} \equiv 1$. Let $S_{t}:=\left\{\exp _{x}(t \nu(x)): x \in \Sigma\right\}$. Pick $t_{0} \in(-\delta, \delta)$ and $x_{0} \in \Sigma$ and set $w_{0}:=w\left(x_{0}, t_{0}\right)$. Then $\Sigma_{t_{0}}$ and $S_{w_{0}}$ are both totally geodesic and touch at $\exp _{x_{0}}\left(t_{0} \nu\left(x_{0}\right)\right)$. Therefore, they coincide. In particular, $w$ is a function of $t$ only, and, since by Proposition 1 . $\int_{\Sigma}(w(\cdot, t)-t) d A=0$, we have $w(x, t) \equiv t$. It follows that $\nu_{t}(x)=\frac{\partial}{\partial t} f_{t}(x)$ and that $\rho_{t} \equiv 1$.

\section{ACKNOWLEDGEMENT}

This research was partially supported by a Warwick Postgraduate Research Scholarship (WPRS).

\section{REFERENCES}

[1] Michael T. Anderson, On area-minimizing hypersurfaces in manifolds of nonnegative curvature, Indiana Univ. Math. J. 32 (1983), no. 5, 745-760, DOI 10.1512/iumj.1983.32.32049. MR711865 (85e:53077)

[2] Lars Andersson, Mingliang Cai, and Gregory J. Galloway, Rigidity and positivity of mass for asymptotically hyperbolic manifolds, Ann. Henri Poincaré 9 (2008), no. 1, 1-33, DOI 10.1007/s00023-007-0348-2. MR2389888 (2009e:53054)

[3] Hubert Bray, Simon Brendle, and Andre Neves, Rigidity of area-minimizing two-spheres in three-manifolds, Comm. Anal. Geom. 18 (2010), no. 4, 821-830. MR2765731 (2012a:53067)

[4] Mingliang Cai, Volume minimizing hypersurfaces in manifolds of nonnegative scalar curvature (Baltimore, MD, 1999), Adv. Stud. Pure Math., vol. 34, Math. Soc. Japan, Tokyo, 2002, pp. 1-7. MR1925731 (2003f:53104)

[5] Mingliang Cai and Gregory J. Galloway, Rigidity of area minimizing tori in 3-manifolds of nonnegative scalar curvature, Comm. Anal. Geom. 8 (2000), no. 3, 565-573. MR.1775139 (2001j:53051)

[6] Jeff Cheeger and Detlef Gromoll, The splitting theorem for manifolds of nonnegative Ricci curvature, J. Differential Geometry 6 (1971/72), 119-128. MR0303460 (46 \#2597)

[7] Doris Fischer-Colbrie and Richard Schoen, The structure of complete stable minimal surfaces in 3-manifolds of nonnegative scalar curvature, Comm. Pure Appl. Math. 33 (1980), no. 2, 199-211, DOI 10.1002/cpa.3160330206. MR562550(81i:53044) 
[8] G. W. Gibbons, Some comments on gravitational entropy and the inverse mean curvature flow, Classical Quantum Gravity 16 (1999), no. 6, 1677-1687, DOI 10.1088/02649381/16/6/302. MR 1697098 (2000j:53089)

[9] Gerhard Huisken and Tom Ilmanen, The inverse mean curvature flow and the Riemannian Penrose inequality, J. Differential Geom. 59 (2001), no. 3, 353-437. MR.1916951 (2003h:53091)

[10] Ernst Heintze and Hermann Karcher, A general comparison theorem with applications to volume estimates for submanifolds, Ann. Sci. École Norm. Sup. (4) 11 (1978), no. 4, 451470. MR.533065 (80i:53026)

[11] I. Nunes, Rigidity of area-minimizing hyperbolic surfaces in three-manifolds, J. Geom. Anal. 23 (2013), no. 3, 1290-1302. MR3078354

[12] R. Schoen and Shing Tung Yau, Existence of incompressible minimal surfaces and the topology of three-dimensional manifolds with nonnegative scalar curvature, Ann. of Math. (2) 110 (1979), no. 1, 127-142, DOI 10.2307/1971247. MR.541332 (81k:58029)

[13] Ying Shen and Shunhui Zhu, Rigidity of stable minimal hypersurfaces, Math. Ann. 309 (1997), no. 1, 107-116, DOI 10.1007/s002080050105. MR1467649 (98g:53113)

[14] James Simons, Minimal varieties in riemannian manifolds, Ann. of Math. (2) 88 (1968), 62-105. MR0233295 (38 \#1617)

Mathematics Institute, University of Warwick, Coventry, CV4 7AL, United Kingdom E-mail address: M.J.Micallef@warwick.ac.uk

Mathematics Institute, University of Warwick, Coventry, CV4 7AL, United Kingdom

E-mail address: V.Moraru@warwick.ac.uk 